\title{
DER STURZ DES DÄDALUS: WOLF BIERMANNS REZEPTION DES MYTHOS VON DÄDALUS UND IKARUS
}

\section{Bernd Seidensticker}

Berlin-Brandenburgische Akademie der Wissenschaften, Jägerstraße, 22/23, 10117, Berlin, Deutschland; bs1@zedat.fu-berlin.de

In the long history of the reception and transformation of the story about Daedalus and Icarus it was the fall of Icarus that more and more became the center of the artistic attention, whereas Daedalus was almost completely sidelined. This also holds true for the reception of the myth in art and literature of the German Democratic Republic. An interesting exception is the poet and song writer Wolf Biermann, who in a famous ballad stylized himself as Prussian Icarus, but after his expatriation from the GDR in 1976 identified himself with Dädalus.

Keywords: Daedalus, Wolf Biermann, reception of the Daedalus and Icarus myth, antiquity in modern poetry, GDR.

Die Geschichte von Dädalus und Ikarus gehört zu den wirkmächtigsten Stoffen der Antike, und wie so oft ist es die Gestaltung Ovids, die die Rezeption des Mythos bis zum heutigen Tag inspiriert und prägt ${ }^{1}$. Ovid hat die Geschichte gleich zweimal ausführlich erzählt ${ }^{2}$ : in der Ars amatoria (2, 22-96) und in den Metamorphosen (8, 183-235). Der unterschiedliche Kontext, in den sie integriert ist, führt zu kleinen Variationen. Im Kern sind die beiden Versionen jedoch identisch:

Nach dem Bau des Labyrinths bittet Dädalus den kretischen König Minos, an dessen Hof in Knossos er lebt, nach Athen zurückkehren zu dürfen. Als der König sich weigert, den berühmtesten Baumeister, Erfinder und Künstler seiner Zeit ziehen zu lassen, beschließt Dädalus zu fliehen. Er baut für sich und seinen kleinen Sohn Ikarus aus Vogelfedern, Leinen und Wachs zwei Paar Flügel, und beim Start warnt er den Sohn, nicht zu hoch und nicht zu tief zu fliegen: „Halte dich auf mittlerer Bahn!“ Zunächst geht alles gut. Dann aber findet der Knabe Gefallen am Fliegen; er wird erst mutiger, dann kühner, schließlich leichtsinniger und fliegt immer höher hinauf. Als er der Sonne zu nahe kommt, schmilzt das Wachs, das die Federn zusammenhält, die Flügel lösen sich auf, und Ikarus stürzt ins Meer, das fortan seinen Namen trägt ${ }^{3}$.

${ }^{1}$ Der Mythos von Dädalus und Ikarus ist zwar zweifellos alt: Schon Homer kennt den berühmten Baumeister Dädalus (Il. 18, 591f.), und seit dem 6. Jahrhundert ist der Stoff auch bildlich auf Vasen und Gemmen, Wandgemälden und Reliefs bezeugt. Aber alle poetischen Gestaltungen und mythographischen Erzählungen des Stoffs zwischen Homer und Ovid sind verloren oder nur ganz bruchstückhaft erhalten. So lassen sich keine sicheren Aussagen darüber machen, ob bzw. in welcher Weise z.B. die zahlreichen Dramen, die für das 5. und 4. Jahrhundert bezeugt sind, oder hellenistische Variationen des Stoffs Ovid beeinflußt haben. Lit. zum Mythos: Robert 1901, 1994-2006; Heeg 1914, 985-988; Frontisi-Ducroux 1975; Nyenhuis 1986, 313-321 (s. v. Daidalos et Ikaros); zur Geschichte der Rezeption des Mythos: Rudd 1988, 21-35; 37-53.

2 Dazu kommt noch die Anspielung auf den Mythos in den Tristien 3, 8, 5-16.

${ }^{3}$ In den Metamorphosen hat Ovid eine Geschichte angehängt $(8,236-259)$, die den Sturz des Ikarus nicht nur als Folge seines jugendlichen Ungehorsams und Übermuts, sondern auch als Bestrafung des Vaters erscheinen läßt: Dädalus - so Ovid - hat vor Jahren den hochbegabten Sohn seiner Schwester aus Furcht, daß dieser ihn mit seinen Erfindungen übertreffen könnte, kopfüber von der Akropolis gestürzt. Doch Athene fängt ihn auf und verwandelt den Knaben, der den Namen Perdix trägt, in eine Wachtel (lateinisch

(C) St. Petersburg State University, 2016 
In Ovids Gestaltung des Mythos ist Dädalus eindeutig der wichtigere Akteur und das war, soweit wir sehen können, auch in den griechischen Texten und Bildern der Fall ${ }^{4}$ und bleibt so bis in die Spätantike.

Besonders beliebt war die Geschichte von Dädalus und Ikarus - wie die Anthologie in der Mythos-Reihe des Reclam Verlags ${ }^{5}$ zeigt - in der ersten Phase ihrer abendländischen Rezeptionsgeschichte, in Renaissance und Barock. Im Anschluß an den Ovid Moralisé, eine weitverbreitete moralisierende Deutung der Metamorphosen, interpretieren die zahlreichen literarischen und bildlichen Darstellungen des Stoffs und die Emblemata mit ihrer Verbindung von Wort und Bild Flug und Sturz des Ikarus als pädagogischmoralische Warnung. „Hochmut kommt vor den Fall“ oder „Verwegenheit groß Schaden bringt", lauten die Urteile, und der ovidische Gedanke, daß Ikarus „,in heißer Begierde nach dem Himmel“ ... ,zu hoch“ hinaufstrebt, kann auch christlich gewendet werden ${ }^{6}$.

Bereits in dieser ersten fruchtbaren Phase der Rezeption der Geschichte beginnt die Reduktion des Stoffs auf eine Episode der mythischen Erzählung. Der kretische Kontext - Minos und Pasiphae, der Minotaurus und das Labyrinth, Gefangenschaft und Flucht - Spielen keine Rolle mehr. Mehr noch, auch die Reduktion auf Ikarus deutet sich an. Zwar ist der Vater in der Rolle des genialen Erbauers der Flügel und des weisen Warners noch präsent ${ }^{7}$, der Schwerpunkt verschiebt sich aber bereits deutlich auf den scheiternden Sohn, und in der weiteren Rezeptionsgeschichte treten auch der Vater und seine Warnungen immer stärker in den Hintergrund. Was bleibt sind Flug und Sturz des Ikarus. Karl-Heinz Stierle hat im Zusammenhang mit dem immer wieder zu beobachtenden Phänomen der Reduktion der ursprünglich bunten mythischen Geschichten auf ein einziges Element von „Schwundstufen des Mythos“ gesprochen ${ }^{8}$. Das gilt z. B. für die Geschichte vom Raub der Europa, von der nichts geblieben ist als „das Mädchen auf dem Stier“, oder für Sisyphus, der nur noch als 'Mann mit dem Stein' weiterlebt'.

Im 18. und 19. Jahrhundert hat der Mythos von Dädalus und Ikarus offenbar seine inspirierende Wirkung auf Dichter und bildende Künstler weitgehend verloren. Daß Goethe in beiden Teilen des Faust den Vater und seinen Sohn als mythische Folie für Faust und für seinen mit Helena gezeugten Sohn Euphorion evoziert, ist die Ausnahme, die die Regel bestätigt. Erst mit dem Beginn der Moderne gewinnt die Geschichte neues Leben ${ }^{10}$.

perdix), die eingedenk des Sturzes die Höhe fürchtet, nur nahe am Boden hinfliegt und ihr Nest nicht auf Bäumen, sondern in Büschen und Hecken baut. Die Wachtel - so schließt Ovid seine Erzählung - freut sich über den Tod des Ikarus und den Schmerz des Vaters.

${ }^{4}$ Es ist wahrscheinlich, daß die stärkere Betonung des Ikarus, wie sie sich z. B. bei Horaz findet (c. 2, 20 und 4, 2) in hellenistischer Zeit begonnen hat.

${ }^{5}$ Aurnhammer, Martin, 1998.

${ }^{6}$ So wie Dante den neugierigen Odysseus im fernen Westen, jenseits der Säulen des Herkules, scheitern läßt und seinen maßlosen Wissensdrang mit den Feuern des Inferno bestraft, so konnte auch Ikarus' Höhenflug als Bestrafung für die Überschreitung der dem Menschen von Gott gesetzten Grenzen verstanden werden.

7 Viele bildliche Darstellungen zeigen Dädalus, wie er die Flügel baut oder wie er sie seinem Sohn anlegt; auf dem Flug zeigen sie ihn meist vor dem Sohn (als Wegweiser) oder unter ihm (als der vorsichtigere).

8 Stierle 1971, 465.

9 Dabei muß festgehalten werden, daß die radikale Reduktion oder besser Komprimierung der Geschichten nicht etwa Verarmung bedeutet, sondern Polyvalenz. Die entstandenen Chiffren können, wie z. B. Sisyphus, aber auch Ikarus zeigen, in immer neuen Kontexten und mit immer neuen Interpretationen für politische und gesellschaftliche, für philosophische, theologische und poetologische Feststellungen und Fragen fruchtbar gemacht werden.

${ }^{10}$ Ein wechselvolles Auf- und Ab ist in der Rezeptionsgeschichte antiker Mythen nichts Ungewöhnliches. 
Zur Revitalisierung haben zweifellos Charles Baudelaires Gedicht „Die Klagen des Ikarus" (aus dem Jahre 1862) ${ }^{11}$ und Pieter Brueghels Gemälde Landschaft mit dem Sturz des Ikarus, das zahlreiche künstlerische Reaktionen auf das Bild und seinen Stoff inspiriert hat, ${ }^{12}$ beigetragen. Einzelne literarische oder bildliche Gestaltung können jedoch nur dann zur Revitalisierung eines Mythos führen, wenn dieser das Lebensgefühl der Zeit trifft, wenn er zur Chiffre für fundamentale kulturelle, gesellschaftliche und politische Entwicklungen und Probleme, zum Bild für weitverbreitete Utopien und Hoffnungen, Sorgen und Ängste der Zeit wird. Und genau dies trifft auf den Dädalus und Ikarus Mythos zu.

Das liegt zunächst einmal daran, daß der uralte Traum des Menschen, sich wie ein Vogel in die Lüfte zu erheben, und der damit verbundene Albtraum des tödlichen Absturzes durch die technischen Entwicklungen der Moderne - durch die Erfindung des Flugzeugs und die begonnene Eroberung des Weltraums - im 20. Jahrhundert große Aktualität gewonnen hat. Es gilt aber noch in einem viel allgemeineren Sinne: Die spannungsreiche Verbindung von Kühnheit und Maßlosigkeit, stürmischem Aufbruch und kläglichem Sturz, Streben und Gefährdung läßt diese Gestalt zu einem einzigartigen Sinnbild der Moderne werden, in der Intelligenz, experimentelle Neugier und Risikobereitschaft zu einem ungeahnten zivilisatorischen Höhenflug geführt, zugleich aber auch nie da gewesene Gefährdungen und Katastrophen gebracht haben.

Schon zu Beginn des Jahrhunderts stehen in expressionistischen und futuristischen Texten die Begeisterung über die Fortschritte des Fliegens und radikale Zivilisationskritik gegenüber und nach dem 2. Weltkrieg wird das ambivalente Bild des abstürzenden Himmelsstürmers neben Prometheus und Sisyphus zu einer der wichtigsten mythischen Chiffren der Moderne.

Die Revitalisierung ist aber eine Revitalisierung der Schwundstufe des Mythos: Im Zentrum stehen eindeutig Ikarus und sein Sturz; Dädalus spielt keine große Rolle ${ }^{13}$.

Das gilt auch (und in ganz besonderem Maße) für die Deutsche Demokratische Republik (DDR), wo Ikarus so intensiv rezipiert worden ist, daß man zu recht von einer Schlüsselgestalt gesprochen hat. Der Erfurter Kunsthistoriker Peter Arlt hat in seiner un-

Viele mythische Geschichten bzw. Figuren verschwinden auf Dauer oder doch vorübergehend; andere können plötzlich auf kürzere oder längere Zeit neues Leben gewinnen.

11 Das Gedicht ist u. a. von Stefan George und Rainer Maria Rilke übersetzt worden. Hier - und in weiteren Texten von Stefan George, Gabriele d'Annunzio und Gottfried Benn - wird Ikarus zum Bild des Dichters, der die Fesseln des Gewohnten zerreißt, die mittlere Bahn verläßt, nach neuen Wegen sucht, und dabei das Äußerste riskiert, auch wenn er daran scheitern muß. - Andere, berühmtere Beispiele für die Revitalisierung eines Mythos durch eine individuelle literarische Gestaltung sind James Joyces Ulysses, der zweifellos zur Bedeutung des Odysseus-Mythos in der Literatur der Moderne beigetragen hat, oder Camus' Sisyphus, der den Sisyphus-Mythos für die Moderne wiederbelebt hat, der viele Jahrhunderte hindurch relativ wenig Beachtung gefunden hatte.

12 Das Bild wird 1912 entdeckt und schnell so berühmt, daß es immer neue Interpretationen und zahlreiche künstlerische Gestaltungen inspiriert hat. In der Reclam-Anthologie Ikarus (s. o. Anm. 5) finden sich nicht weniger als 15 Texte auf das Gemälde von W.H. Auden bis Brecht, und das ist nur eine Auswahl.

${ }_{13}$ Eine berühmte Ausnahme ist James Joyce, der die deutlich autobiographisch geprägte Hauptfigur seiner ersten beiden Romane Stephen Dedalus genannt hat. - Das Motto für Portrait of the Artist as a Young Man lautet: et ignotas animum dimittit in artes (Met. 8, 186: über den Bau der Flügel) und der Roman endet mit dem Satz: Alter Vater, alter Künstler, steh ' mir bei, jetzt und immer. - Dieser Stephen Dedalus alias James Joyce erweist sich allerdings eher als ein Ikarus, der zwar nicht abstürzt, aber auch nicht wirklich hoch fliegen kann. Joyce selber fühlte sich offenbar mit Dädalus als Künstler, aber auch durch Exil und Wanderschaft verbunden. - Dädalus spielt zudem vor allem in der intensiven Rezeption des Minotaurosmythos eine Rolle. 
publizierten Habilitationsschrift Zur Antikerezeption in der bildenden Kunst der DDR aus dem Jahre $1987^{14}$ gezeigt, daß keine andere Gestalt der antiken Mythologie die Phantasie ostdeutscher Künstler stärker inspiriert hat als Ikarus ${ }^{15}$, und diese Feststellung gilt in kaum geringerem Maße für die ostdeutschen Dichter ${ }^{16}$. Neben die zahlreichen Variationen des Sturzes im Werk der berühmten Leipziger Maler Bernhard Heisig und Wolfgang Mattheuer (und viele andere Darstellungen des Themas) treten eine lange Reihe in erster Linie lyrischer Texte. Vor allem Günter Kunert hat Jahrzehnte lang immer neue IkarusGedichte geschrieben ${ }^{17}$.

Dädalus ist zwar nicht ganz vergessen, bleibt jedoch ohne größere Wirkung auf bildende Künstler und Dichter ${ }^{18}$. Eine berühmte Ausnahme ist Wolf Biermann, dessen Identifikation mit Dädalus deswegen besonders interessant ist, weil er auch den wohl bekanntesten Ikarustext der DDR geschrieben hat: Das Lied vom Preußischen Ikarus

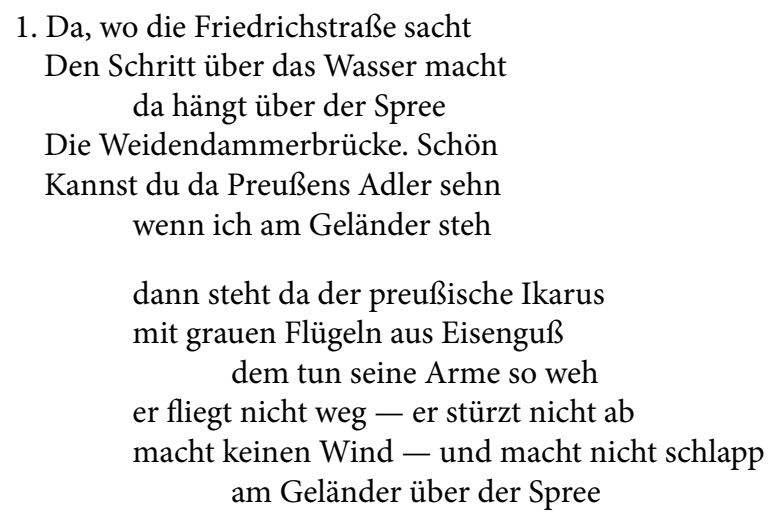

2. Der Stacheldraht wächst langsam ein Tief in die Haut, in Brust und Bein ins Hirn, in graue Zelln Umgürtet mit dem Drahtverband Ist unser Land ein Inselland umbrandet von bleiernen Welln da steht der preußische Ikarus mit grauen Flügeln aus Eisenguß dem tun seine Arme so weh er fliegt nicht weg - und stürzt nicht ab macht keinen Wind - und macht nicht schlapp am Geländer über der Spree

${ }^{14}$ Mehr als 20 Jahre später hat Arlt auf der Basis seiner Sammlung ein Buch zum selben Thema publiziert: Arlt 2008, zu Ikarus: 114-126.

${ }_{15}$ Am berühmtesten sind die immer neuen Variationen des Themas durch die Leipziger Maler Bernhard Heisig und Wolfgang Mattheuer.

${ }^{16}$ Von den zahlreichen Texten, die wir im Berliner Archiv für Antikerezeption in der deutschsprachigen Literatur der Gegenwart gesammelt haben, stammt der größte Teil aus der DDR.

17 Vgl. Seidensticker, Wessels 2004, 255-271.

18 Daneben sind mir aus der DDR-Literatur nur noch eine Reihe von Dädalus-Gedichten bekannt: Gosse 1975, 23-25; Rosenlöcher 1982, 24-25; Kunert 1990, 43; 2002, 149. 
3. Und wenn du wegwillst, mußt du gehn

Ich hab schon viele abhaun sehn

aus unserem halben Land

Ich halt mich fest hier, bis mich kalt

Dieser verhaßte Vogel krallt

und zerrt mich übern Rand

dann bin ich der preußische Ikarus

mit grauen Flügeln aus Eisenguß

dann tun mir die Arme so weh

dann flieg ich hoch - dann stürz ich ab

mach bißchen Wind - dann mach ich schlapp

am Geländer über der Spree.

Die 1975 entstandene Ballade ist ein Lied über die Ausbürgerung aus der DDR, die Wolf Biermann in dieser Zeit drohte. Der Liedermacher sieht sich selbst als preußischen Ikarus, präsentiert sich jedoch in einer paradoxen Korrektur des Mythos ${ }^{19}$ als einen Ikarus, der gar nicht fliegen will.
er fliegt nicht weg - er stürzt nicht ab
macht keinen Wind und macht nicht schlapp
am Geländer über der Spree.

Auch wenn er in einem Gefängnis lebt, das langsam ein Teil von ihm wird, auch wenn er sich wie auf einer isolierten Insel fühlt, die von bleiernen Wellen umspült wird, auch wenn viele andere das „Inselland“ DDR verlassen haben und verlassen werden: er will nicht fliegen, nicht fliehen; will festhalten an seiner Hälfte Deutschlands und seinen sozialistischen Träumen ${ }^{20}$, bis der preußische Adler, eben noch Symbol des Ich, nun Symbol des Regimes, ihn „übern Rand zerrt“. Dann wird er fliegen müssen. Biermann weiß, daß sein Flug von Ost und West ein „bißchen Wind“ machen wird, aber er glaubt und fürchtet, daß er nach kurzem Flug abstürzen wird.

In seinem Essay Der Sturz des Dädalus aus dem Jahre $1990^{21}$ erklärt Biermann den Anlaß des Liedes und seinen Titel. Bei einem Besuch von Allen Ginsburg zeigte Biermann dem amerikanischen Dichter 'sein Berlin' und stellte sich, als die Beiden die Friedrichstraße hinauf über die Weidendammer Brücke gingen, demonstrativ vor den Adler aus Gußeisen. der die Mitte der Brücke krönt:

„Schau, Allen, wenn ich mich so richtig hinstelle, dann wachsen mir die Flügel des verfluchten Vogels aus den Schultern. Dann bin ich der preußische Ikarus“. Ginsburg photographierte Biermann in dieser Pose und die Beiden beschlossen, jeder ein Gedicht bzw. ein Lied über diese „hochalberne Szene ${ }^{\text {“22 }}$ zu schreiben.

19 Zum Begriff der Mythenkorrektur cf. Seidensticker, Vöhler, Emmerich 2005. Zu einer paradoxalen Form der Mythenrezeption: 1-18.

${ }^{20}$ In dem etwas später entstandenen Lied Und als wir ans Ufer kamen heißt es: Was wird bloß aus unseren Träumen / In diesem zerrissenen Land / Die Wunden wollen nicht zugehn / Unter dem Drecksverband / Und was wird aus unseren Freunden / Und was noch aus dir, aus mir - Ich möchte am liebsten weg sein / Und bleibe am liebsten hier - am liebsten hier.

${ }^{21}$ Biermann 1990, 289-312.

22 Vorworte: Biermann 1978, 107. 
So 'hochalbern' die Szene auch gewesen sein mag, so bedeutsam und prophetisch war der Text, der daraus entstand, für den Liedermacher. Als Biermann 1976, mehr als zehn Jahre nach dem totalen Auftrittsverbot ${ }^{23}$, überraschend die Erlaubnis erhält, die Einladung der westdeutschen Gewerkschaft IG Metall zu einer Konzertreise durch die Bundesrepublik Deutschland anzunehmen, singt er gleich beim ersten Konzert, am 13. November in der Kölner Sporthalle, natürlich auch „die Ballade von der Angst vor einem Absturz in den Westen ${ }^{\text {"24, }}$, und die Reaktion läßt nicht lange auf sich warten. Nur drei Tage später ,zerrt der verhaßte Vogel", wie es im Lied heißt, ihn tatsächlich „übern Rand“: Er wurde „wegen grober Verletzung der staatsbürgerlichen Pflichten“ ausgebürgert. Und das „bißchen Wind", das Biermann prophezeit

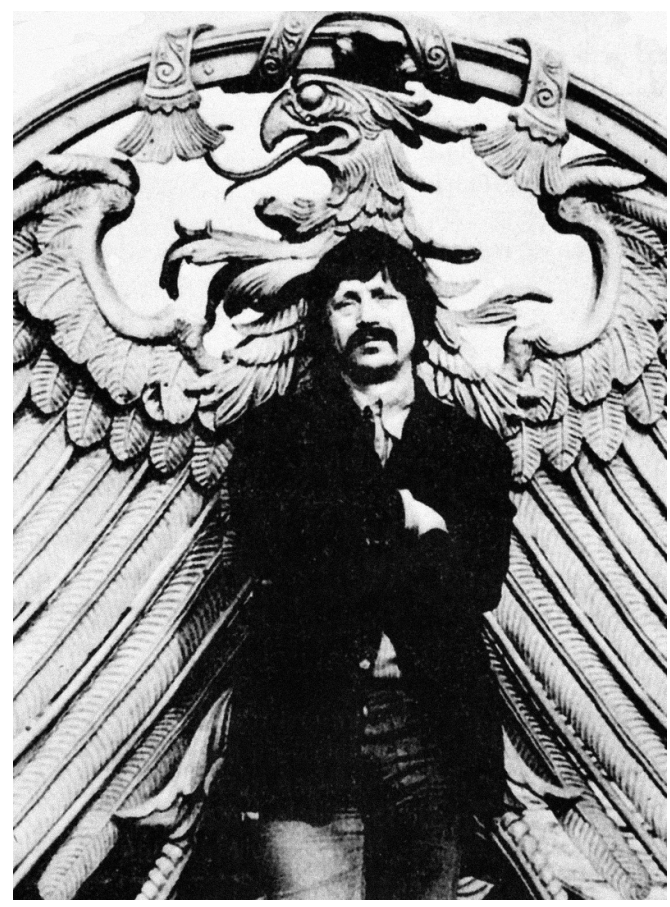
hatte, entwickelte sich zu einem veritablen Sturm: Zahlreiche ostdeutsche Künstler protestierten gegen die Entscheidung, und viele von ihnen verließen in der Folge die DDR ${ }^{25}$.

Eine Prophezeiung des Textes wurde nicht wahr: Biermann 'machte nicht schlapp', auch wenn er die Furcht vor dem 'schlapp machen' weiter thematisierte. Im Vorwort der zweiten Sammlung, die er im Westen publizierte, heißt es mit ausdrücklichem Rückblick auf die Ballade vom Preußischen Ikarus. „Falls ich im Westen leben müßte, das wußte ich,

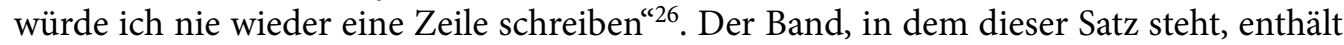
jedoch nicht nur die „letzten Osttexte“, sondern auch die „ersten Westtexte“27. Als Titel dieser zurück- und vorausblickenden Sammlung alter und neuer Lieder wählt Biermann noch seine Selbstbestimmung als Preußischer Ikarus ${ }^{28}$, aber bereits im Vorwort stellt er sich und seinen Lesern die Frage: „Warum wurde Ikarus so viel berühmter als sein Vater? Warum ist nicht der Vater tiefer eingekerbt in unser Gedächtnis, der es doch geschafft hat? Daedalus hatte den guten Einfall mit den künstlichen Flügeln, Daedalus baute sie aus Adlerfedern und Bienenwachs. Und Daedalus war klug, er flog nicht zu tief am Wasser, nicht zu hoch an der Sonne und schaffte so die Flucht von der Insel Kreta über das Meer, in dem der Sohn ertrank. Dummkopf, der auf den Vater nicht hören wollte. Oder was?“29

${ }^{23}$ Das Verbot erfolgte auf dem 11. Sitzung des ZK der Sozialistischen Einheitspartei Deutschlands (SED) im Jahre 1965.

24 Biermann 1990, 294.

25 Literatur zur Ausbürgerung: Berbig 1994; Liebermann 2006, 993-1003; Grünbaum 1976.

26 Biermann 1978, 107-108.

27 Die erste Sammlung nach der Ausbürgerung ist noch ausdrücklich als „Nachlass“, d. h. als Ende der Produktion, bestimmt: Der Titel des 1977 erschienenen Bandes heißt Nachlaß 1.

28 Biermann 1978, 107-108.

${ }^{29}$ Biermann 1978, 107. 
An dieser Stelle taucht die Überlegung ganz unvermittelt auf, und erst am Ende des Essays wird deutlicher, was Biermann bewegt. Die Frage bleibt aber auch hier ohne explizite Antwort ${ }^{30}$.

Erst viele Jahre später hat Biermann in dem Essay mit dem programmatischen Titel Der Sturz des Dädalus ${ }^{31}$ detailliert expliziert, was Dädalus symbolisiert und was er für ihn selbst bedeutet. Der Text beginnt mit einer Interpretation von Brueghels Landschaft mit dem Sturz des Ikarus ${ }^{32}$. Die wohl von allen Betrachtern des berühmten Gemäldes geteilte Verwunderung darüber, daß Brueghel ganz darauf verzichtet hat, auch den Vater des ins Meer stürzenden Ikarus darzustellen, läßt Biermann erneut die Frage stellen, warum „nicht Daidalos, der geflügelte Vater, ein geflügeltes Wort geworden ist, wohl aber Ikarus, sein entflügelter Sohn" ${ }^{\text {“33 }}$, und seine eigene Entscheidung für Ikarus erklären: „Als ich jünger war, ergriff mich das Schicksal des Jungen. Ikarus, der Traum vom Fliegen. Wenn ich ein Vöglein wär ${ }^{34}$. Die Mauer, der Stacheldraht, die Bluthunde - die Phantasien. Und die Angst vor dem Absturz in den Westen. Damals fragte ich nicht nach dem Alten“ (292).

Jetzt nach dem „Absturz in den Westen“ interessiert ihn der Alte. Er verweist auf die wohl eine Generation später entstandene Kopie des Brueghel-Gemäldes ${ }^{35}$, auf dem auch Dädalus zu sehen ist, „klein, hoch oben, links, grad, wie er fliegt, aber kehrt nicht um, er rührt keinen Flügel mehr für den Sohn" (292), und nun identifiziert er sich mit dem weiterfliegenden Vater: „Dieser Dädalus, den meine ich. Sein Sturz stachelt mich im schönen Altona an der Elbe. Der Sturz dessen, der nicht stürzte. Der Sturz ins banale Weiterleben. Ich rede mal wieder von mir, will sagen von dir: Der Sturz des Dädalus“(292). Nach einem Rückblick auf den Preußischen Ikarus und eine erneute Formulierung seiner Angst, keine Lieder mehr schreiben zu können ${ }^{36}$, formuliert er das neue Credo: „Als ich jung war, starb ich täglich am Liebeskummer mit dem Kommunismus. Jetzt im Alter leb ich davon. Will sagen: Das einstmals ikarische Leben verdädaliert sich. Nicht der attraktive Tod, sondern das Weiterleben ohne die glänzenden Wunden des Märtyrers vorn an der Rampe" (296f.).

Am Ende des Essays gewinnt die persönliche Absage an Ikarus auch gesellschaftliche Bedeutung. Biermann erinnert seine Leser an den utopistischen Roman Voyage en Icarie von Étienne Cabet, aus dem das Wort Kommunismus stammt. Cabet habe das Land seiner kommunistischen Träume „nach dem Kleindarsteller im Dädalus-Drama“ Ikarien genannt; Biermann wünscht sich ein anderes Buch: „Ich kann es mir nicht selber

${ }^{30}$ Ib. 128: „Wie kommt es, daß wir immer den unerhörten Fall brauchen, um uns in unserer grauen Normalität zu erkennen? ... Ich weiß nicht warum Ikarus statt Dädalus, ich weiß nicht, warum die Weidendammer Brücke und nicht die Friedrichstraße?“

31 Biermann 1990.

32 Es gibt von Biermann auch ein Gedicht auf das Gemälde von Marc Chagall Der Sturz des Ikarus im Musée National d'Art Moderne, Centre Georges Pompidou, Paris; das Gedicht trägt den Titel: Großes Rot bei Chagall, in Biermann 1982, 48.

${ }^{33}$ Im weiteren Verlauf des Essays kommt er immer wieder auf diese Frage zurück.

${ }^{34}$ Biermann zitiert den Anfang des bekannten Volkslieds Wenn ich ein Vöglein wär, das zuerst in Johann Gottfried Herders Sammlung Stimmen der Völker in Liedern (Erstes Buch, Nr. 12) erschienen ist.

35 Die zweite Version, die Dädalus in der linken Bildhälfte hoch am Himmel zeigt, tauchte 1935 auf; sehr wahrscheinlich ist auch das 1912 aufgetauchte Gemälde (s. o. Anm. 12) nicht das Original, sondern eine Kopie.

36 Biermann 1990, 296: „Schluß mit dem Schreiben. Vom Westen wußte ich nichts - und vom Osten wollte ich nichts mehr wissen. ... Ich glaubte nicht, daß ich im Westen noch brauchbare Lieder zustande bringe. Der Osten hatte mich gestachelt, der Westen, das wußte ich, würde mich stumpfen“. 
schreiben - aber ein Buch, das ich heute gern lesen würde, hätte den Titel: Die Reise nach Dädalus-Land“.

Der preußische Ikarus ist zum Dädalus geworden: „Meine Hoffnung heißt Dädalus, der Kunstfertige, der Schuldbeladene ${ }^{37}$, der Erfindungsreiche. Mit ihm will ich mich und meine Freunde vergleichen, und an ihm messe ich die Staatsmänner meiner Zeit" $(310)^{38}$.

Ganz, freilich mag Biermann sich denn doch nicht von Ikarus trennen. Er zitiert das bekannte Volkslied Wenn ich ein Vöglein wär ${ }^{39}$ und erklärt: „Ja, den Traum vom Fliegen, das Ikarische, will ich nie nicht verlernen - weils aber nicht kann sein / bleib ich halt hier" $(312)^{40}$ Aber das ist nicht sein letztes Wort. Der Essay schließt mit den Worten: „Es ist eben wie's ist. Und ich finde mich nicht damit ab. Mich versöhnt mit den Verhältnissen, daß ich sie ändern will. Und solches Träumen austreiben wird mir kein dunkler Tag, keine helle Nacht" (312).

Der Mythos vom Baumeister Dädalus und seine Interpretation durch den großen Liedermacher Wolf Biermann, könnten, denke ich, dem Empfänger der Festschrift gefallen, der selber ein großer, erfindungsreicher Baumeister ist und der auch wenn manche Träume 'abgestürzt' sind, immer weitergeflogen ist und weiterfliegt! Dafür, lieber Alexander, vielen Dank und alles Gute!

\section{Literaturhinweise}

Arlt P. Die Flucht des Sisyphos. Griechischer Mythos und Kunst. Eine europäische Bildtradition, ihre Aktualität in der DDR und heute. Kunstverlag Gotha, 2008.

Aurnhammer A., Martin D. (Hgg.) Mythos Ikarus. Texte von Ovid bis Wolf Biermann. Leipzig, Reclam, 1998.

Berbig, R.u. a. (Hrsg.) In Sachen Biermann. Protokolle, Berichte und Briefe zu den Folgen einer Ausbürgerung. Berlin, Ch. Links, 1994.

Biermann W. Der Sturz des Dädalus. Klartexte im Getümmel. 13 Jahre im Westen. Von der Ausbürgerung bis zur November-Revolution. Hg. von H. Stein. Köln, Kiepenheuer und Witsch, 1990, 289-312.

Biermann W. Großes Rot bei Chagall. Biermann W. Verdrehte Welt - das seh ich gerne, Köln, Kiepenheuer und Witsch, 1982, 48.

Biermann W. Preußischer Ikarus. Lieder, Balladen, Gedichte, Prosa. Köln, Kiepenheuer und Witsch, 1978.

Frontisi-Ducroux F. Dédale. Mythologie de l'Artisan en Grèce ancienne. Paris, Fr. Maspéro, 1975.

Gosse P. Munterung an Dädalus. Ortungen - Gedichte und Notate. Halle, Mitteldeutscher Verlag, 1975, 23-25.

Grünbaum R. Wolf Biermann 1976. Die Ausbürgerung und ihre Folgen. Erfurt, Landeszentrale für politische Bildung Thüringen, 2011.

Heeg, J. Ikaros 2. RE. IX 1, 1914, 985-988.

Kunert G. Daidalos. Fremd daheim. Gedichte. München, Wien, Hanser, 1990, 43.

${ }^{37}$ Biermann thematisiert ausführlich Dädalus' Ermordung seines Neffen Perdix und die Bestrafung durch Athene.

38 Als Beispiel nennt er Michael Gorbatschow. Im folgenden heißt es: „Mit den durchmischten Tugenden des Dädalus werden wir zwar nicht, wie Ikarus, in den Höhen des Mythos und im Gedächtnis der Menschen glänzend überleben, vielleicht aber auf der platten Erde“. (310) und: „Ich glaube die Menschheit hat nur in der Tradition des Dädalus eine Chance zu überleben. Ikarische Sturzflüge kann keiner gebrauchen“ (311).

39 Biermann hat auch eine Variation des Volkslieds mit dem Titel Folkslied geschrieben. Die letzte Strophe des Lieds, das in dem Band Affenfels und Barrikade, Köln 1986, 87 erschienen ist, endet mit einer weiteren Absage an den Preußischen Adler Ikarus: Meleken, wenn ich ein Vöglein wär / würde ich fliegen mit dir noch mal / wie es im alten Liedchen heißt / meintwegen Ikarus, Dädalus auch / wäre ich Spottdrossel, Möwe und Loon / aber nie nicht kein Preußen-Adler / und auch kein deutsches Suppenhuhn.

${ }^{40}$ Die zitierten Worte sind die letzten beiden Zeilen der ersten Strophe des Volkslieds: Wenn ich ein Vöglein wär / und auch zwei Flüglein hätt, / flög ich zu dir, / weil's aber nicht kann sein, / bleib ich allhier. 
Kunert G. Dädalus' Erbe. So und nicht anders. Ausgewählte und neue Gedichte. Mit einem Nachwort von Michael Krüger. München; Wien, Hanser, 2002, 149.

Liebermann D. Im traurigen Monat November war's. Interview mit Wolf Biermann über die Ausbürgerung und die Folgen. Deutschland Archiv, 2006, 39/6, 993-1003.

Nyenhuis J.E. Daidalos et Ikaros. LIMC. 3, 1986, 313-321.

Robert C. Daidalos. RE. IV, 2, 1901, 1994-2006.

Rosenlöcher Th. Dädalus. Ich lag im Garten bei Kleinzschachwitz. Gedichte und zwei Notate. Halle, Leipzig, Mitteldeutscher Verlag, 1982, 24-25.

Rudd N. Daedalus and Icarus (i): from Rome to the End of the Middle Ages; Daedalus and Icarus (ii): From the Renaissance to the Present Day. Ovid Renewed. Ovidian Influences on Literature and Art from the Middle Ages to the Twentieth Century. Ed. by Ch. Martindale. Cambridge, CUP, 1988, 21-35; 37-53.

Seidensticker, B., Vöhler, M. Emmerich W.Zum Begriff der Mythenkorrektur. Mythenkorrekturen. Zu einer paradoxalen Form der Mythenrezeption. Berlin, de Gruyter, 1995, 1-18.

Seidensticker B., Wessels A. (Hgg.) Kunerts Antike. Eine Anthologie. Freiburg, Rombach, 2004.

Stierle K. H. Mythos als 'Bricolage' und zwei Endstufen des Prometheusmythos. M. Fuhrmann (Hg.) Terror und Spiel. Probleme der Mythenrezeption. München, Fink, 1971 (Poetik und Hermeneutik 4), 455-472.

For citation: Bernd Seidensticker. Der Sturz des Dädalus: Wolf Biermanns Rezeption des Mythos von Dädalus und Ikarus. Philologia Classica 2016, 11(1), 89-97. DOI: 10.21638/11701/spbu20.2016.109

\section{ПАДЕНИЕ ДЕДАЛА: РЕЦЕПЦИЯ МИФА О ДЕДАЛЕ И ИКАРЕ У ВОЛЬФА БИРМАНА}

Бернд Зейденштикер

В течение долгой истории рецепции и трансформации мифа о Дедале и Икаре падение Икара все более оказывалось в центре внимания художников, тогда как Дедал был почти полностью отодвинут на второй план. В этом отношении рецепция мифа в ГДР не представляет собой чего-то необычного. Интересным исключением оказался, однако, Вольф Бирман, который в известной балладе стилизовал себя в качестве прусского Икара, но позже, после изгнания из ГДР, стал отождествлять себя с Дедалом.

Ключевые слова: Дедал, Вольф Бирман, рецепция мифа о Дедале и Икаре, античность в современной поэзии, ГДР. 